\title{
A new therapeutic strategy with istradefylline for postural deformities in Parkinson's disease
}

\author{
Shinsuke Fujioka ${ }^{1,2}$, Ryoko Yoshida ${ }^{3}$, Kanako Nose $^{2}$, Yuka Hayashi ${ }^{2}$, Takayasu Mishima $^{1}$, Jiro Fukae ${ }^{1}$, \\ Kosuke, Kitano ${ }^{4}$, Hitoshi Kikuchi ${ }^{2}$, Yoshio Tsuboi ${ }^{2}$ \\ ${ }^{1}$ Department of Neurology, Murakami Karindoh Hospital, Fukuoka, Japan \\ ${ }^{2}$ Department of Neurology, Fukuoka University School of Medicine, Fukuoka, Japan \\ ${ }^{3}$ Department of Internal Medicine, Murakami Karindoh Hospital, Fukuoka, Japan \\ ${ }^{4}$ Department of Rehabilitation, Murakami Karindoh Hospital, Fukuoka, Japan
}

\begin{abstract}
Aim of the study. Postural deformities are common in Parkinson's disease (PD) patients. Several treatment options have been reported, but responses to these treatments appear unpredictable. Istradefylline is a novel drug for PD. Cases of PD patients whose postural deformities were improved after withdrawal of dopamine agonists and initiation of istradefylline are presented.

Materials and methods. Four consecutive patients with postural deformities including antecollis, Pisa syndrome, and camptocormia were recruited and treated with istradefylline in combination with withdrawal of dopamine agonists, which are possible causes of postural deformities.

Results. The dopamine agonists were discontinued an average of 26 months after the development of the postural deformities, and istradefylline was initiated an average of 1.3 months after dopamine agonist withdrawal. Three patients with preserved paraspinal muscle volume showed good responses to the treatment regimen at least two months after dopamine agonist withdrawal.

Conclusions and clinical implications. Postural deformities caused by dopamine agonists generally improve less than two weeks after dopamine agonist withdrawal. Given the response time in the present study, the response was unlikely to be caused solely by dopamine agonist withdrawal. Istradefylline can be a potential therapeutic option; however, appropriate selection of patients for treatment with istradefylline is warranted.
\end{abstract}

Key words: Parkinson's disease, istradefylline, postural deformity, camptocormia, antecollis, Pisa syndrome

(Neurol Neurochir Pol 2019; 53 (4): 291-295)

\section{Introduction}

Patients with Parkinson's disease (PD) often develop postural deformities including camptocormia, antecollis, Pisa syndrome, and scoliosis [1]. Possible pathophysiological mechanisms for postural deformities, such as dystonia, rigidity, myopathy, impaired proprioception, spine structural changes, and impaired spatial perception, have been proposed [1]. Postural deformities can be induced by several drugs, including amantadine, levodopa, and valproic acid. Dopamine agonists can also induce postural deformities, and withdrawal of the dopamine agonist alone may lead to prompt symptom relief. However, withdrawal of the dopamine agonist may worsen the motor symptoms of PD. Other treatment options including deep brain stimulation and botulinum toxin injection are available, but they are unsatisfactory in some patients [2]. Istradefylline, an adenosine A2A receptor antagonist, is a novel non-dopaminergic drug available for the treatment of

Address for correspondence: Yoshio Tsuboi, Department of Neurology, Fukuoka University, 7-45-1, Nanakuma, Johnan-ku, Fukuoka-city, Fukuoka 8140180, Japan, Phone: +81-92-801-1011 ext. 3525, e-mail: tsuboi@cis.fukuoka-u.ac.jp 
PD in Japan [3]. A clinical trial of istradefylline for PD patients showed reduced off time and improved Unified Parkinson's Disease Rating Scale motor scores. However, a 12-week, double-blind study to assess the efficacy of istradefylline failed to prove efficacy in improving motor symptoms [4]. The detailed pharmacokinetics of istradefylline are unknown.

Very recently, Suzuki et al. reported that nine of $18 \mathrm{PD}$ patients with postural abnormalities who were treated with istradefylline showed a significant improvement $(p<0.05)$ in posture, as assessed using the sub-score for posture on the Movement Disorder Society revision of the Unified Parkinson's Disease Rating Scale part III [5]. Possible mechanisms underlying the response to the therapy have been previously suggested [6]. Postural deformity can be caused by dystonia, and blockade of $\mathrm{A} 2 \mathrm{~A}$ receptors was shown to restore the impairment of synaptic plasticity in dystonia mice. Another potential mechanism is that an inadequate dose of anti-parkinsonian drugs could be one of the causes of postural deformity that can be ameliorated by istradefylline, which can decrease the off-periods [7].

However, that study did not clarify which factors affect response. In addition, the clinical details of each patient were not described in that report. The cases of four PD patients with postural deformities who were treated with istradefylline in combination with dopamine agonist withdrawal are here presented. Three patients showed improvements in postural deformities without deterioration of motor function. The details of the patients' clinical disease courses are presented, along with the possible predictors of postural improvement with istradefylline.

\section{Materials and methods}

The clinical charts of 103 PD patients who visited the Department of Neurology at our hospital between 1 April, 2015 and 31 March, 2016 were reviewed, and four PD patients with postural deformities were identified. Treatment with istradefylline was then begun after obtaining their informed consent for treatment. The clinical diagnosis of PD was made by board-certified neurologists (SF, TM, JF, YT) according to the UK Brain Bank Clinical Diagnostic Criteria [8]. Patients with previous spinal bone fractures were excluded, as were patients who had mild postural deformities that did not impact upon daily life. Data on demographics and clinical and magnetic resonance imaging (MRI) findings for patients treated with istradefylline were retrieved.

All experimental procedures were conducted in accordance with the policies and ethical principles of the Declaration of Helsinki. This study was approved by the Ethics Committee of our institution.

\section{Results}

The patients' mean age at $\mathrm{PD}$ onset and mean disease duration were 69 (standard deviation (SD) 7.0) years and
5 (SD 0.5) years, respectively. The mean Hoehn and Yahr stage was approximately 3 . The detailed clinical courses of the four patients are presented below.

Patient 1 developed clumsiness in the upper right limb at 73 years of age. She was diagnosed with PD and started on ropinirole at 74 years, with amelioration of her symptoms. She began to take levodopa at 76 years. She showed a forward-bent posture that had gradually worsened, and she also developed a dropped head during the disease course (Fig. 1A). Zonisamide and selegiline were added during the disease course, but the postural deformity did not respond to the medication. Neurological examination showed camptocormia and antecollis. Ropinirole was withdrawn, and istradefylline $(40 \mathrm{mg} /$ day) was simultaneously started at 77 years. Two months after the initiation of istradefylline, the postural abnormalities gradually improved, and she showed normal posture for an elderly person three months after beginning istradefylline (Fig. $1 \mathrm{~B}, \mathrm{C})$. MRI showed good preservation of paraspinal muscle volume (Fig. 1D-G).

Patient 2 presented with a gait disturbance at 72 years of age and was diagnosed with PD at 74 years. She started taking levodopa, and pramipexole was subsequently added, which improved her parkinsonism considerably. She developed a dropped head at 74 years (Fig. 1H). Selegiline and entacapone were added at 74 and 75 years, respectively. Pramipexole was changed to ropinirole at 76 years. Botulinum toxin injection for the sternocleidomastoid muscles did not improve her symptoms at the age of 77 years. On neurological examination, she had camptocormia and antecollis, and ropinirole was discontinued at 79 years. Two months after discontinuation of ropinirole, istradefylline $(40 \mathrm{mg} /$ day) was started. Two months after the initiation of istradefylline, postural abnormalities showed gradual improvement, and normal posture for an elderly person was restored three months after beginning istradefylline (Fig. 1I, J). MRI showed mild paraspinal muscle atrophy (Fig. 1K-N).

Patient 3 developed a tremor in the upper right limb at 62 years of age. She was diagnosed with PD at 63 years. She was first given levodopa, which ameliorated her parkinsonism. She started to take rotigotine and amantadine during the course of the illness. She developed lateral trunk flexion at 66 years, with gradual deterioration (Fig. 1O). On examination, she showed Pisa syndrome. Rotigotine was withdrawn, and zonisamide and trihexyphenidyl were added, but the symptoms did not improve. One month after withdrawal of rotigotine, istradefylline ( $40 \mathrm{mg} /$ day) was started. Six months after initiating istradefylline, her postural abnormalities were mildly improved (Fig. 1P). MRI showed mild atrophy of the paraspinal muscles (Fig. 1Q-T).

Patient 4 developed a resting tremor and motor slowness in the lower left limb at 59 years. She was diagnosed with PD at 60 years, and she started taking levodopa and pramipexole, which improved her symptoms. She presented with a dropped head at 67 years. Despite adjustment of anti-parkinsonian drugs, parkinsonism and the dropped head gradually 

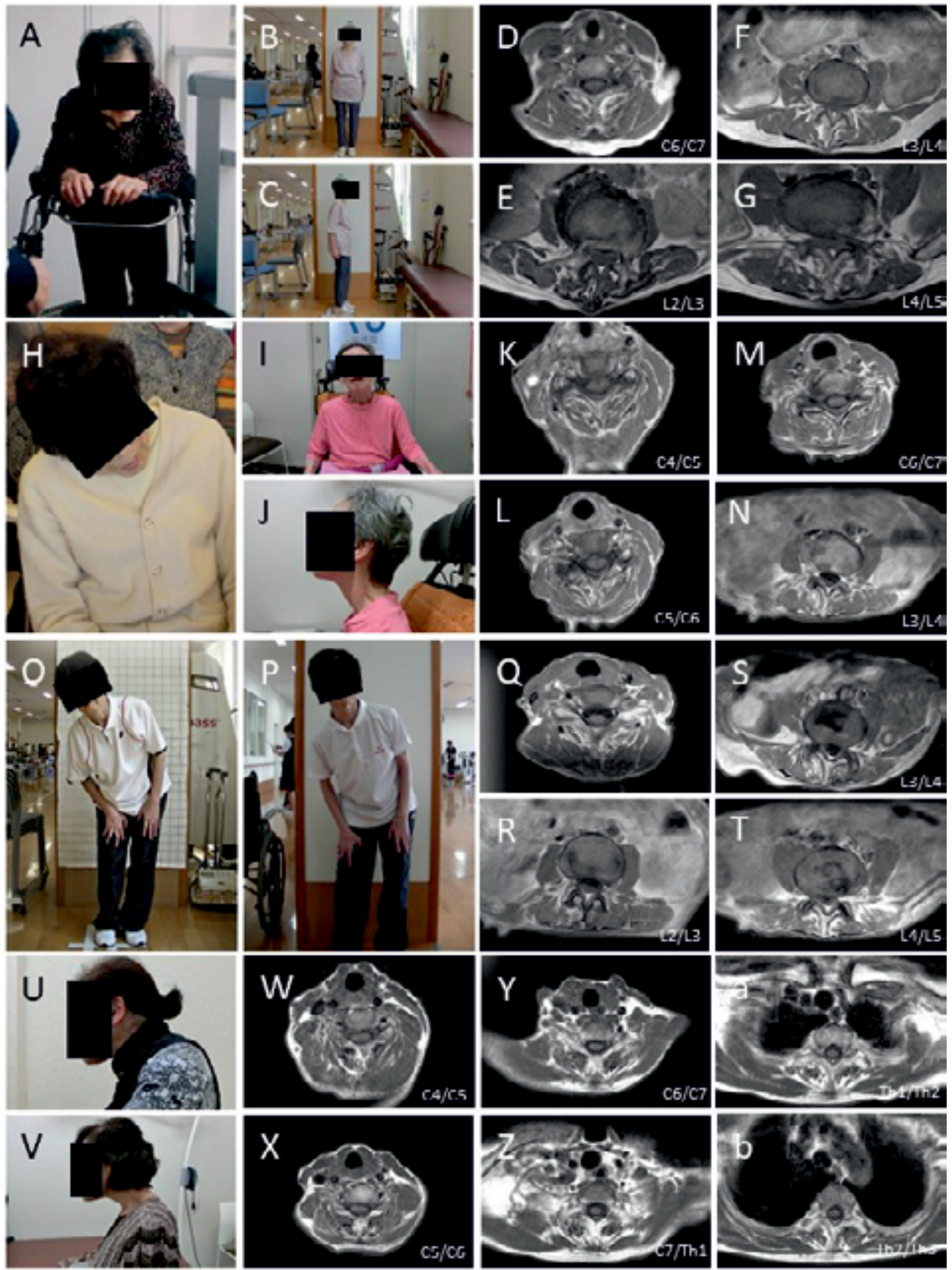

Figure 1. Description of patients before and after initiation of istradefylline and MRI observations.

Patient 1 presented with camptocormia and antecollis (A), which improved greatly after initiation of istradefylline (B, C). MRI T1WI (D-G) shows well-preserved paraspinal muscle volume. Patient 2 showed antecollis $(\mathrm{H})$ that improved greatly three months after initiation of istradefylline $(\mathrm{I}, \mathrm{J})$. Patient 3 showed Pisa syndrome (0), which was partially ameliorated after initiation of istradefylline (P). MRI T1WI examinations of both patients (K-N: Patient 2; Q-T: Patient 3) show relatively preserved paraspinal muscle volume. Patient 4 showed antecollis (U) and experienced no improvement after initiation of istradefylline (V). MRI T1WI examination of Patient 4 (W-Z, a, b) shows moderate atrophy of the paraspinal muscles

worsened (Fig. 1U). On neurological examination, she had antecollis; the pramipexole was reduced, and istradefylline was started at 70 years. Pramipexole was withdrawn two months before starting istradefylline ( $40 \mathrm{mg} /$ day). However, even eight months after initiation of istradefylline, the dropped head had not improved (Fig. 1V). MRI (T1WI) showed moderate atrophy of the paraspinal muscles (Fig. 1W-Z, 1a, 1b).
The demographic and clinical data of the patients are set out in Table 1.

\section{Discussion}

The clinical courses of four PD patients with postural deformities who were treated with istradefylline in combination 
Table 1. Summary of demographic and clinical data of four PD patients with postural deformities

\begin{tabular}{|c|c|c|c|c|c|c|c|c|c|}
\hline Patient & $\begin{array}{c}\text { AAO } \\
\text { (years) }\end{array}$ & $\begin{array}{c}\text { DD } \\
\text { (years) }\end{array}$ & $\begin{array}{l}\text { H\&Y } \\
\text { stage }\end{array}$ & $\begin{array}{l}\text { Type of } \\
\text { postural } \\
\text { deformities }\end{array}$ & $\begin{array}{l}\text { Combined medica- } \\
\text { tions before initiation } \\
\text { of istradefylline (/day) }\end{array}$ & $\begin{array}{l}\text { Period A } \\
\text { (months) }\end{array}$ & $\begin{array}{l}\text { Period B } \\
\text { (months) }\end{array}$ & $\begin{array}{l}\text { Period C } \\
\text { (months) }\end{array}$ & $\begin{array}{l}\text { Period D } \\
\text { (months) }\end{array}$ \\
\hline 1 & 73 & 4 & 3 & $\begin{array}{l}\text { Camptocor- } \\
\text { mia, } \\
\text { antecollis }\end{array}$ & $\begin{array}{l}\text { Levodopa } 300 \mathrm{mg} \\
\text { Ropinirole } 6 \mathrm{mg} \\
\text { Zonisamide } 25 \mathrm{mg} \\
\text { Selegiline } 2.5 \mathrm{mg}\end{array}$ & 8 & 12 & 0 & 2 \\
\hline 2 & 72 & 7 & 4 & Antecollis & $\begin{array}{l}\text { Levodopa } 600 \mathrm{mg} \\
\text { Ropinirole } 8 \mathrm{mg} \\
\text { Droxidopa } 600 \mathrm{mg}\end{array}$ & 6 & 60 & 2 & 2 \\
\hline 3 & 62 & 4 & 3 & $\begin{array}{l}\text { Pisa syndro- } \\
\text { me }\end{array}$ & $\begin{array}{l}\text { Levodopa } 200 \mathrm{mg} \\
\text { Rotigotine } 18 \mathrm{mg} \\
\text { Amantadine } 100 \mathrm{mg}\end{array}$ & 10 & 6 & 1 & 6 \\
\hline 4 & 59 & 11 & 3 & Antecollis & $\begin{array}{l}\text { Levodopa } 450 \mathrm{mg} \\
\text { Pramipexole } 8 \mathrm{mg} \\
\text { Zonisamide } 25 \mathrm{mg}\end{array}$ & 84 & 36 & 2 & NA \\
\hline
\end{tabular}

AAO - age at onset; DD - disease duration; H\&Y - Hoehn \& Yahr; NA - not applicable;

Period A - period between initiation of dopamine agonist and onset of postural deformities;

Period $B$ - period between onset of postural deformities and withdrawal of dopamine agonist

Period C - period between withdrawal of dopamine agonist and initiation of istradefylline;

Period D - period between initiation of istradefylline and improvement of postural deformities

with the withdrawal of dopamine agonists are here presented. Patient 1 showed camptocormia and antecollis, Patient 2 showed antecollis, Patient 3 showed Pisa syndrome, and Patient 4 had antecollis. All patients had initially been treated with levodopa/carbidopa and a dopamine agonist before the development of postural deformities. One of the most common causes of postural deformities seen in PD is medications, especially dopamine agonists. Thus, the dopamine agonists were stopped, and the patients were treated with istradefylline during the disease course to avoid deterioration of motor function. For Patient 1, istradefylline was started at the same time as dopamine agonist withdrawal. For the other patients, istradefylline was initiated at least two months after dopamine agonist withdrawal. Three of the four PD patients showed clinical improvement of postural deformities at least two months after dopamine agonist withdrawal. Postural deformities caused by dopamine agonist medications generally improve relatively quickly ( $\leq 2$ weeks after dopamine agonist withdrawal). Given the response time seen in the presented patients, the clinical response was unlikely to have been caused solely by dopamine agonist withdrawal. In addition, a very recent report by Suzuki et al. showed improvements in postural deformities with istradefylline treatment [5].

Paraspinal muscle volume is considered to predict improvement of postural deformities by deep brain stimulation. Saki et al. performed subthalamic deep brain stimulation in $14 \mathrm{PD}$ patients with camptocormia and showed significantly higher paraspinal muscle volume in patients who responded well to therapy compared to those who showed partial or no response
[9]. In the presented cases, MRI showed preservation of paraspinal muscle volume in Patients 1-3, who showed good to mild improvement with istradefylline, whereas Patient 4, who showed no clinical improvement, showed moderate atrophy of the paraspinal muscles. As for deep brain stimulation, paraspinal muscle volume is a potentially good predictor of success when treating postural deformities in PD with istradefylline.

The exact pharmacokinetics of adenosine A2A receptor blockade remain unclear. Dystonia is one cause of postural deformities. Napolitano et al. reported that adenosine A2A receptor blockade rescues physiological synaptic plasticity, leading to an improved phenotype in DYT1 mutant mice [10]. The pedunculo-pontine tegmental nucleus is associated with muscle tone control. Another potential mechanism is that adenosine A2A receptor blockade by istradefylline inhibits basal ganglia output through an indirect pathway that leads to amelioration of excess inhibition of this nucleus [11].

One limitation of this study was the absence of quantitative evaluation of postural deformities. Another limitation is the absence of strategic methods for MRI acquisition to assess muscle volume. In addition, due to the retrospective nature of this study, some clinical information was missing.

\section{Clinical implications/future directions}

Postural deformities greatly impair patients' activities of daily living and unquestionably lead to worsening of their quality of life; therefore, novel therapies are needed. Given the limited availability of pharmacological agents for postural 
deformities, istradefylline is a potential therapeutic option. Dopamine agonist withdrawal may lead not only to an improvement in postural deformity, but also to a deterioration of parkinsonism. Conversion from a dopamine agonist to istradefylline is potentially safe and beneficial for patients with postural deformities, without leading to deterioration of motor symptoms.

\section{Acknowledgement}

The authors would like to thank the staff at Fukuoka University Hospital for their kind support and for the management of patients.

Funding: This publication was prepared without any external sources of funding.

Conflict of interest: None.

\section{Author contributions}

All authors contributed substantially to this study: conception and design of the study (S.F., Y.T.); acquisition and interpretation of data (S.F., R.Y., K.N., Y.T.); drafting of the article (S.F.); revising the article critically for important intellectual content (R.Y., K.N., H.Y., T.M., J.F., K.K., H.K., Y.T.); and final approval of the version to be submitted (S.F., R.Y., K.N., H.Y., T.M., J.F., K.K., H.K., Y.T.).

\section{References}

1. Doherty KM, van de Warrenburg BP, Peralta MC, et al. Postural deformities in Parkinson's disease. Lancet Neurol. 2011; 10(6): 538549, doi: 10.1016/S1474-4422(11)70067-9, indexed in Pubmed: 21514890 .
2. Margraf NG, Wrede A, Deuschl G, et al. Pathophysiological Concepts and Treatment of Camptocormia. J Parkinsons Dis. 2016; 6(3): 485501, doi: 10.3233/JPD-160836, indexed in Pubmed: 27314757.

3. Pourcher E, Fernandez HH, Stacy M, et al. Istradefylline for Parkinson's disease patients experiencing motor fluctuations: results of the KW6002-US-018 study. Parkinsonism Relat Disord. 2012; 18(2): 178-184, doi: 10.1016/j.parkreldis.2011.09.023, indexed in Pubmed: 22000279.

4. Fernandez HH, Greeley DR, Zweig RM, et al. 6002-US-051 Study Group. Istradefylline as monotherapy for Parkinson disease: results of the 6002-US-051 trial. Parkinsonism Relat Disord. 2010; 16(1): 16-20, doi: 10.1016/j.parkreldis.2009.06.008, indexed in Pubmed: 19616987.

5. Suzuki K, Miyamoto T, Miyamoto M, et al. Could istradefylline be a treatment option for postural abnormalities in mid-stage Parkinson's disease? J Neurol Sci. 2018; 385: 131-133, doi: 10.1016/j. jns.2017.12.027, indexed in Pubmed: 29406892.

6. Kataoka $\mathrm{H}$, Sugie $\mathrm{K}$. Does istradefylline really have a dystonic mechanism? J Neurol Sci. 2018; 388: 233-234, doi: 10.1016/j. jns.2018.03.020, indexed in Pubmed: 29559176.

7. Mizuno Y, Kondo T. Japanese Istradefylline Study Group. Adenosine A2A receptor antagonist istradefylline reduces daily OFF time in Parkinson's disease. Mov Disord. 2013; 28(8): 1138-1141, doi: 10.1002/mds.25418, indexed in Pubmed: 23483627.

8. Hughes AJ, Daniel SE, Kilford L, et al. Accuracy of clinical diagnosis of idiopathic Parkinson's disease: a clinico-pathological study of 100 cases. J Neurol Neurosurg Psychiatry. 1992; 55(3): 181-184, doi: 10.1136/jnnp.55.3.181, indexed in Pubmed: 1564476.

9. Sakai W, Nakane S, Urasaki E, et al. The Cross-Sectional Area of Paraspinal Muscles Predicts the Efficacy of Deep Drain Stimulation for Camptocormia. J Parkinsons Dis. 2017; 7(2): 247-253, doi: 10.3233/ JPD-160948, indexed in Pubmed: 28157107.

10. Napolitano F, Pasqualetti M, Usiello A, et al. Dopamine D2 receptor dysfunction is rescued by adenosine $A 2 A$ receptor antagonism in a model of DYT1 dystonia. Neurobiol Dis. 2010; 38(3): 434-445, doi: 10.1016/j.nbd.2010.03.003, indexed in Pubmed: 20227500.

11. Takakusaki $\mathrm{K}$, Saitoh $\mathrm{K}$, Harada $\mathrm{H}$, et al. Role of basal ganglia-brainstem pathways in the control of motor behaviors. Neurosci Res. 2004; 50(2): 137 151, doi: 10.1016/j.neures.2004.06.015, indexed in Pubmed: 15380321. 